\title{
SCALE-RESOLVING SIMULATION OF FLOW THROUGH A PERIODIC ARRAY OF CUBES
}

\author{
MICHEL ELKHOURY \& AMINA ELCHEIK \\ Department of Industrial and Mechanical Engineering, Lebanese American University, Lebanon
}

\begin{abstract}
Previous Scale-Resolving Simulation studies of flow over urban-like obstacles uses Large Eddy Simulation and course grids to reduce computational cost. However, the coarser the mesh the more reliance on the subgrid scale model to accurately account for scales associated with high wavenumbers. Furthermore, when high-resolution simulations are of importance, such as the transport of urban contaminants, mesh refinement becomes necessary. Often clustering of mesh cells produce errors at grid-refinement interfaces, mainly on the fine side of the mesh when it is located upstream of the coarse one. Three scale-resolving turbulence models, the One-Equation Scale-Adaptive Simulation (One-Eq.SAS), the Shear Stress Transport-Improved Delayed Detached Eddy Simulation (SST-IDDES), and the Algebraic Wall-Modelled Large Eddy Simulation (WMLES) models are utilized to assess their effect on the accuracy of the results when applied on both coarse and mesh-refined grids. The selection of these models was first based on the computational cost where the WMLES is the cheapest to solve since it involves no partial differential equation, while the SST-IDDES model is computationally the most expensive. Simulations are carried out on a relevant and complex test case of flow through a periodic array of cubes. The results reveal that models that do not inherent grid scale parameters in their formulation perform best in flows with global instabilities.

Keywords: Scale-Resolving Simulation, Scale-Adaptive Simulation, One-Equation model, bluff body simulation.
\end{abstract}

\section{INTRODUCTION}

Turbulence models are still the choice in almost all engineering applications due to their simplicity, low computational cost, and acceptable accuracy. The latter is an issue of concern to many Computational Fluid Dynamics (CFD) users in the engineering society. Surely, in lieu of utilizing these models, Large Eddy Simulation (LES) can accurately predict complex flows with massive separation, vortex shedding, and strong adverse pressure gradient. However, LES requires larger integration time compared to Unsteady Reynolds Average Navier-Stokes (URANS), smaller time-step, and higher mesh resolution. When combining all these effects with a real-life engineering application, it becomes evident that this approach is prohibitive and users generally revert back to URANS for prompt results, not to mention the advancements that have been made to improve the accuracy of turbulence models.

Recently, Menter and Egorov [1] developed the Scale-Resolving Simulation (SRS) concept that is based on the inclusion of the von Karman length scale in their two-equation turbulence $k$ - $\omega$-SST closure. This in turn enables the model to adjust to resolved turbulent structures rather than dissipating them as RANS models do, an ability of the model referred to by Scale-Adaptive Simulation (SAS). These models perform best in globally unstable flows that are associated with massive flow separation, in which they are capable of resolving large- and small-scale turbulent structures [2]. A key feature of the SAS models is that they produce turbulent flow structures similar to LES and DES without suffering from the explicit grid sensitivity and they do not require unsteady inflow condition. The latter is important in internal flow problems where a fully developed profile is imposed at the inlet. SAS models revert back to RANS mode when insufficient spatial and temporal resolution is encountered. 
The main objective of this work is to demonstrate that a simple one-equation turbulence model can accurately simulate a challenging engineering problem such as the flow through a periodic array of cubes. The transformation methodology of this partially lagging oneequation model was originally derived from the $k-\varepsilon$ closure using Bradshaw et al. [3], i.e. $|-\overline{u v}| / k$ a constant turbulent structure parameter. The model operates in SRS mode and thus can resolve turbulent scales in flows with massive separation.

In the present work a partially lagging one-equation turbulence model [4] is applied to a challenging turbulent flow test case and assessed against the SST-IDDES [5] and the WMLES [6] models. Sub-grid modelling accuracy is assessed by a comparison between the low- and the high-density meshes. Unphysical perturbations arising from mesh clustering at interfaces in the streamwise/crossflow direction [7] is also assessed by comparing the results of the three models on regular and clustered grids.

\section{NUMERICAL APPROACH}

The computations throughout this work were performed using the commercial software Fluent 17.2. The one-equation SAS model was solved utilizing the User Defined Scalars (UDS) capabilities that are available in the software. All models were solved using the explicit pressure based solver. Momentum equations were discretized using the second order bounded central difference scheme. This was necessary to minimize numerical dissipation in order to resolve flow structure in the regions dominated with flow separation. The standard interpolation scheme was used in calculating the cell-face pressures. A second order timeaccurate formulation was used in transient computations. In addition, convective terms were handled using second order upwind-based discretization scheme with minimum underrelaxation factors of 0.8 .

\section{RESULTS}

For all considered models, calculations were run fully turbulent and a minimum convergence criterion of $1 \times 10^{-3}$ of scaled residuals of all flow variables was achieved. Furthermore, all computations were performed on the IBM HPC nextScale M5 with 112 cores of Intel(R)Xeon(R)-CPU-E5-2667-v3-@-3.20 GHz running with double precision at 4 Teraflops.

\subsection{Flow past an array of cubes}

This is a challenging three-dimensional test case that could mimics building aerodynamics. Detailed flow measurements using laser Doppler anemometer were carried out by Meinders [8] and Meinders and Hanjalic [9]. A total of 250 cubes were placed in a 2-D plane channel flow in an aligned configuration consisting of 25 rows of 10 cubes each. A cube spacing of $3 H$, where $H$ is the side length of the cube, was set in both the streamwise and spanwise directions with a channel height of $3.4 \mathrm{H}$. A schematic of the array of cubes delineating the computational domain and a side view of a matrix of cubes, showing stations at which the comparison between numerical and experimental results, are shown in Fig. 1 with a $4 H \times 4 H$ $\times 3.4 \mathrm{H}$ in the streamwise, spanwise and wall-normal directions, respectively. The Reynolds number based on the mean bulk velocity of $U_{b}=3.86 \mathrm{~m} / \mathrm{s}$, which was based on the average mass flow rate and surface area normal to the flow direction, and a cube edge of $H=15 \mathrm{~mm}$ was 3,855 . Periodic boundary condition was applied in both the streamwise and spanwise directions whereas a no-slip boundary condition was applied at the top and bottom in the wall-normal direction, as well as at the surface of the cube. It is worth noting that flow measurements were made around the 18th row of the array where a fully developed periodic 

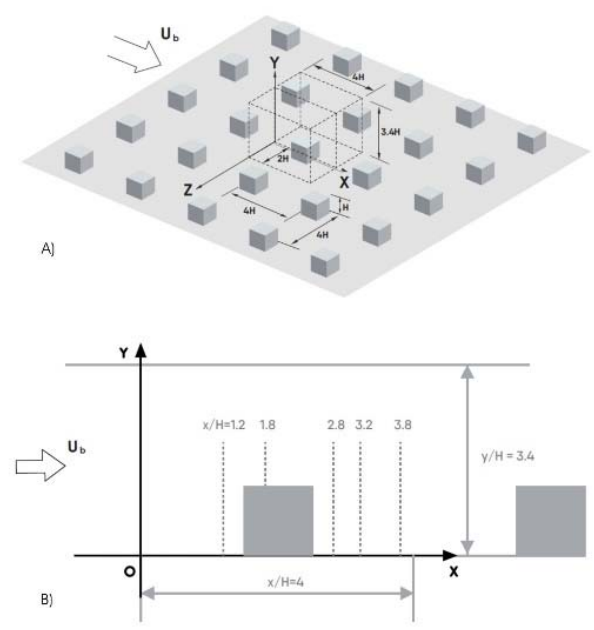

Figure 1: (a) A 3-D isometric view of a matrix of cubes showing the computational domain; (b) A side view of a matrix of cubes in a bounded plan channel showing stations at which numerical and experimental results will be compared.

state was experimentally achieved [9], justifying the use of the periodic boundary condition in the flow direction.

Meshes consisting of $55 \times 45 \times 55$, and $150 \times 180 \times 150$ nodes were used in the computations. Two meshes with the size of the larger grid were constructed, the first had an almost uniform grid spacing throughout the domain while the other had mesh refinement near the surface of the cube as depicted in Fig. 2. Table 1 presents a comparison between the current mesh sizes and turbulence models against those utilized in literature. It is worth to note that a relatively coarse grid sizes were used in literature owing to the fact that such lowdensity grids are desired for the simulation of urban boundary layer. Hence, simulations over the coarse mesh allow the assessment of the models' abilities to accurately account for the sub-grid stresses and associated flow features. In addition, simulations on the denser mesh, i.e. $150 \times 180 \times 150$ nodes, reduce the influence of sub-grid modeling, thereby improving the models' abilities to resolve flow structures. Hence, a comparison between the low- and the high-density grids can shed light on the sub-grid modeling capabilities of the considered models. Errors due to grid-refinement at interfaces, that are not wall-bounded in the streamwise/crossflow direction, are measured by a direct comparison of the results predicted

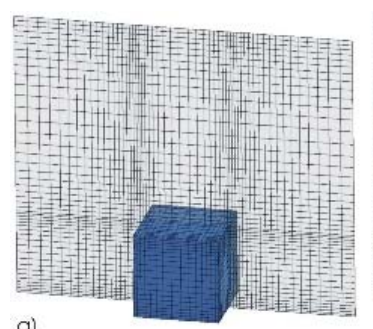

a)

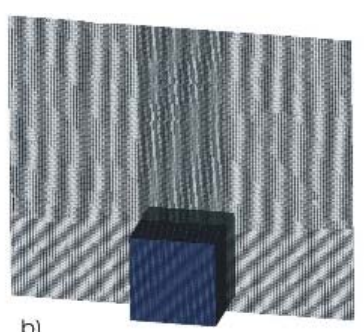

b)

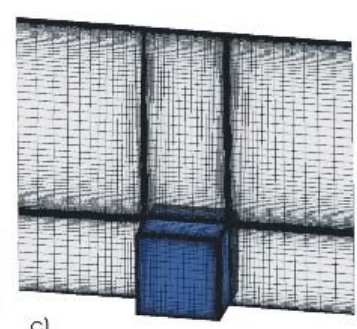

c)

Figure 2: Mesh in the x-y plane that passes through the center of the cube. (a) Low-density mesh; (b) Uniform mesh; and (c) Clustered mesh. 
Table 1: Comparison of models and utilized grids for flow past an array of cubes.

\begin{tabular}{|c|c|c|c|}
\hline Contributor & Model & $\begin{array}{c}\text { Grid, } \\
\mathrm{Nx}, \mathrm{Ny}, \mathrm{Nz}\end{array}$ & $\begin{array}{l}\text { Number of points } \\
\text { on cube side }\end{array}$ \\
\hline Hsieh et al. [10] & $\begin{array}{l}\text { LES - Standard Smogarinsky, } \\
\text { PANS, k- } \varepsilon \text { RANS }\end{array}$ & $49 \times 49 \times 49$ & - \\
\hline Yang et al. [11] & Integral wall-modeled LES & $32 \times 32 \times 32$ & 8 \\
\hline $\begin{array}{l}\text { Goodfriend et al. } \\
{[7]}\end{array}$ & $\begin{array}{l}\text { LES - Standard and mixed } \\
\text { model }\end{array}$ & $84 \times 96 \times 84$ & 28 \\
\hline \multirow{3}{*}{ Present } & \multirow{3}{*}{$\begin{array}{l}\text { WMLES, SST-IDDES, One- } \\
\text { Eq.-SAS }\end{array}$} & $55 \times 45 \times 55$ & 15 \\
\hline & & $150 \times 180 \times 150$ & 70 \\
\hline & & $\begin{array}{c}150 \times 180 \times 150 \\
\text { Clustered }\end{array}$ & 70 \\
\hline
\end{tabular}

on the clustered and the regular unclustered grids. It is worth to note that the level of the unphysical perturbations at grid-refinement interfaces is a direct indicator of the model's success.

The flow was initialized with a $U_{b}$ without any velocity fluctuation in the streamwise direction. For the course and fine meshes, the time step was chosen with a temporal resolution of $\Delta t / T_{b}=1 / 32$ where $T_{b}=H / U_{b}$ is the turnover time. Averaging of flow variables started after the elapse of $500 T_{b}$ to ensure that the final averaged results were independent of the initial flow conditions. The averaging process took place over $200 T_{b}$ to ensure statistical convergence of averaged flow variables. To ensure temporal independent solution, a higher resolution of $\Delta t / T_{b}=1 / 64$ was used on the regular unclustered mesh using the WMLES. Results of averaged velocities and turbulent statistics were almost identical to those obtained at the lower resolution time step. Based on its mathematical formulation, the WMLES model, was expected to require the least computational resources however, it was computationally the most expensive, requiring 17, 23, and 20 iterations per time-step to converge on the unclustered, clustered, and the coarse mesh. The IDDES model on the contrary, was supposed to be the most expensive owing to its complex mathematical formulation. However, the model required 6,1 , and 6 iterations per time-step to converge on the unclustered, clustered, and the coarse mesh, respectively. The One-Eq.-SAS model required 15, 9, and 20 iterations per time-step for the same sequence of grids reported before.

The streamwise velocity profiles plotted in the vertical direction, according to Fig. 1(b), at the centerline of the square $(\mathrm{z} / \mathrm{H}=0$ plane $)$ are shown in Fig. 3. A comparison among models on the coarse and regular unclustered meshes is made in Fig. 3(a), while Fig. 3(b) depicts a comparison on the regular clustered and unclustered meshes. Assessments of all models are made against experimental data. For the regular unclustered mesh, all velocity profiles are very well predicted in both Figs 3(a) and (b). However, all models seem to suffer to a various extent when run on the coarse mesh as depicted in Fig. 3(a) with the One-Eq.-SAS model being closest to experiments. Both the IDDES and WMLES models deviate from experiments when simulations are carried out on the clustered mesh however, the One-Eq.SAS model consistently reproduce velocity profiles with minimal deviations.

Fig. 4 shows the streamwise velocity profiles plotted in the horizontal direction at a plane that corresponds to $y / H=0.5$. It is evident that the One-Eq.-SAS model predicts the closest velocity profiles at all $x / H$ stations on the coarse mesh while the WMLES model performs 

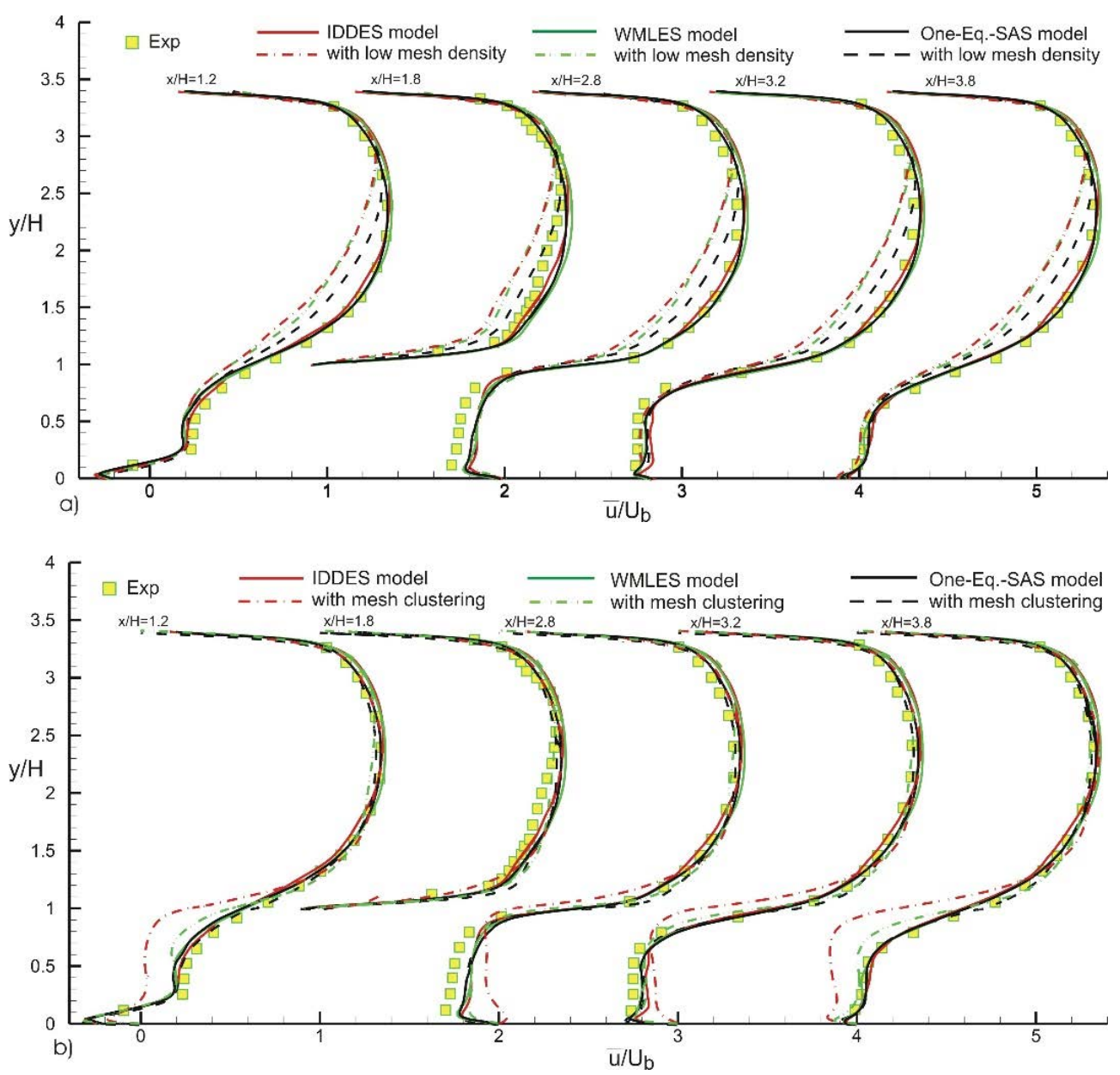

Figure 3: Mean streamwise velocity profiles on the vertical $(x-y)$ plane that cuts through the center of the cube at $z / H=0$ showing effect of (a) mesh density; and (b) mesh clustering. Each successive $x / H$ profile starting from the one at $x / H=1.2$ is offset by one unit from the previous profile.

best on the clustered mesh as depicted in Fig. 4(b). The WMLES predicts profiles that are slightly better than those predicted by the IDDES model on the coarse mesh. The IDDES model underpredicts the streamwise velocity profiles between $1.0<\mathrm{z} / \mathrm{H}<2.0$ when run on the unclustered regular mesh. It also fails to accurately capture near-wall variations of reverse flow on the clustered mesh between $0.0<\mathrm{z} / \mathrm{H}<0.6$ as shown in Fig. 4(b). The WMLES model underpredicts the velocity profile when ran on the clustered mesh at $x / H=3.8$.

The mean spanwise velocity profiles on the $x-z$ plane that cuts midway through the cube at $y / H=0.5$ are depicted in Fig. 5. The IDDES model predicts the least accurate velocity profiles and is closely followed by the WMLES model on all three considered meshes. In the region close to the street canyon between the cubes, i.e. $1.5<\mathrm{z} / H<2.0$ the IDDES model 

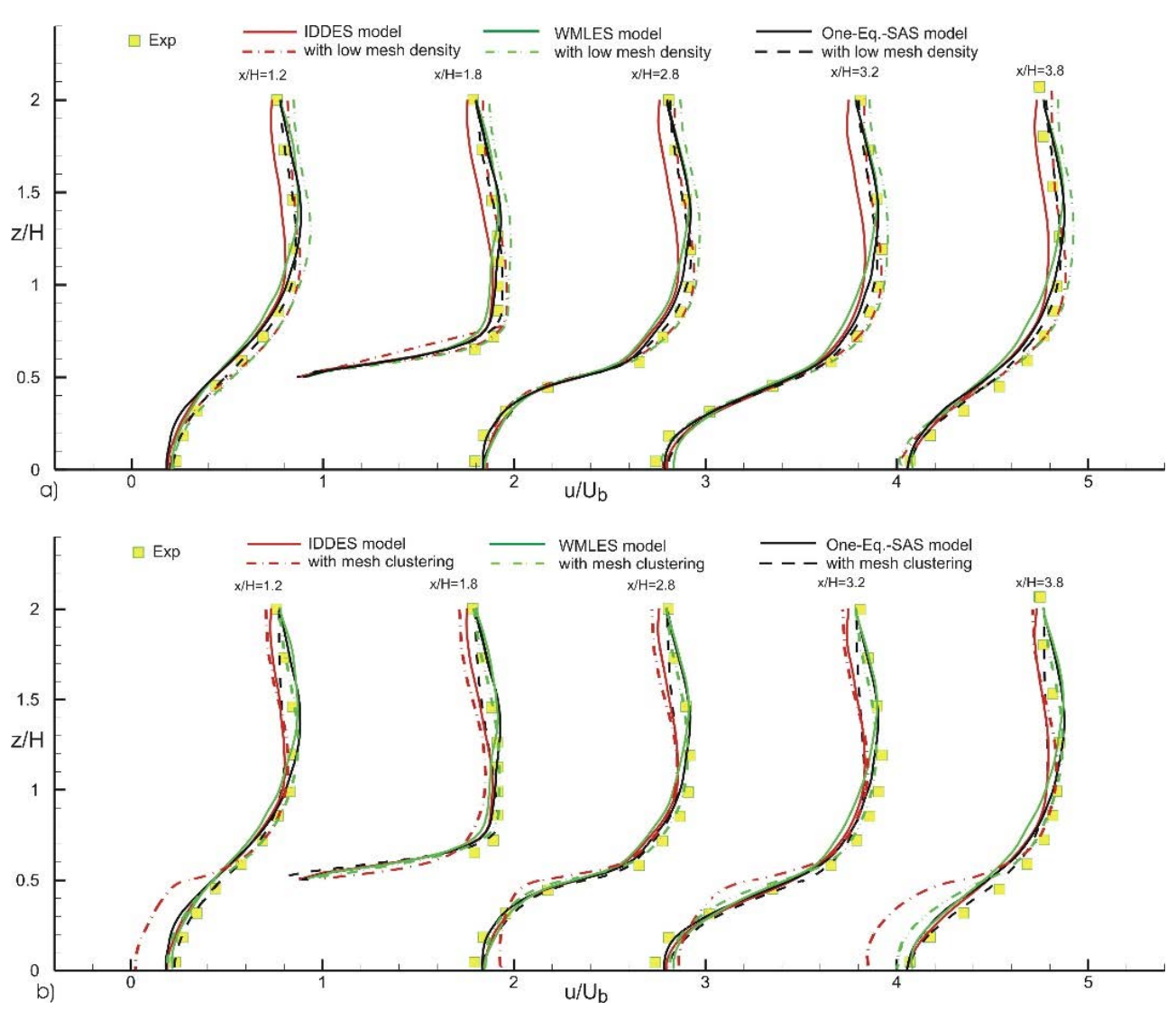

Figure 4: Mean streamwise velocity profiles on the horizontal $(x-z)$ plane that cuts through the center of the cube at $y / H=0.5$ showing effect of (a) mesh density; and (b) mesh clustering. Each successive $x / H$ profile starting from the one at $x / H=1.2$ is offset by one unit from the previous profile.

deviates from the experiments on the regular unclustered mesh. The IDDES model deviates the most amongst other models when grid-refinement mesh is considered as shown in Fig. 5(b). The One-Eq.-SAS model deviates the least when comparisons are made between the regular unclustered mesh and the other two grids. It also predicts acceptable velocity profiles, however, not as accurate as the streamwise velocity profiles that were reported in the previous two figures.

Profiles of streamwise normalized Reynolds normal stress, $\overline{u^{\prime} u^{\prime}} / U_{b}^{2}$, plotted on the $\mathrm{x}-\mathrm{y}$ plane at the centerline of the cubes $(z / H=0)$ are shown in Fig. 6. Peaks in $\overline{u^{\prime} u^{\prime}} / U_{b}^{2}$ are observed to take place around $y / H \approx 1.0$ that are due to the separating thin shear layer from rooftop and sidewalls of the cube. This peak attenuates and spreads in-between the cubes as it gets advected by turbulent transport from the center of its corresponding shear layer. All models acceptably predict the Reynolds normal stress on both the coarse and regular density meshes with the One-Eq.-SAS model being the least accurate in the street canyon region 

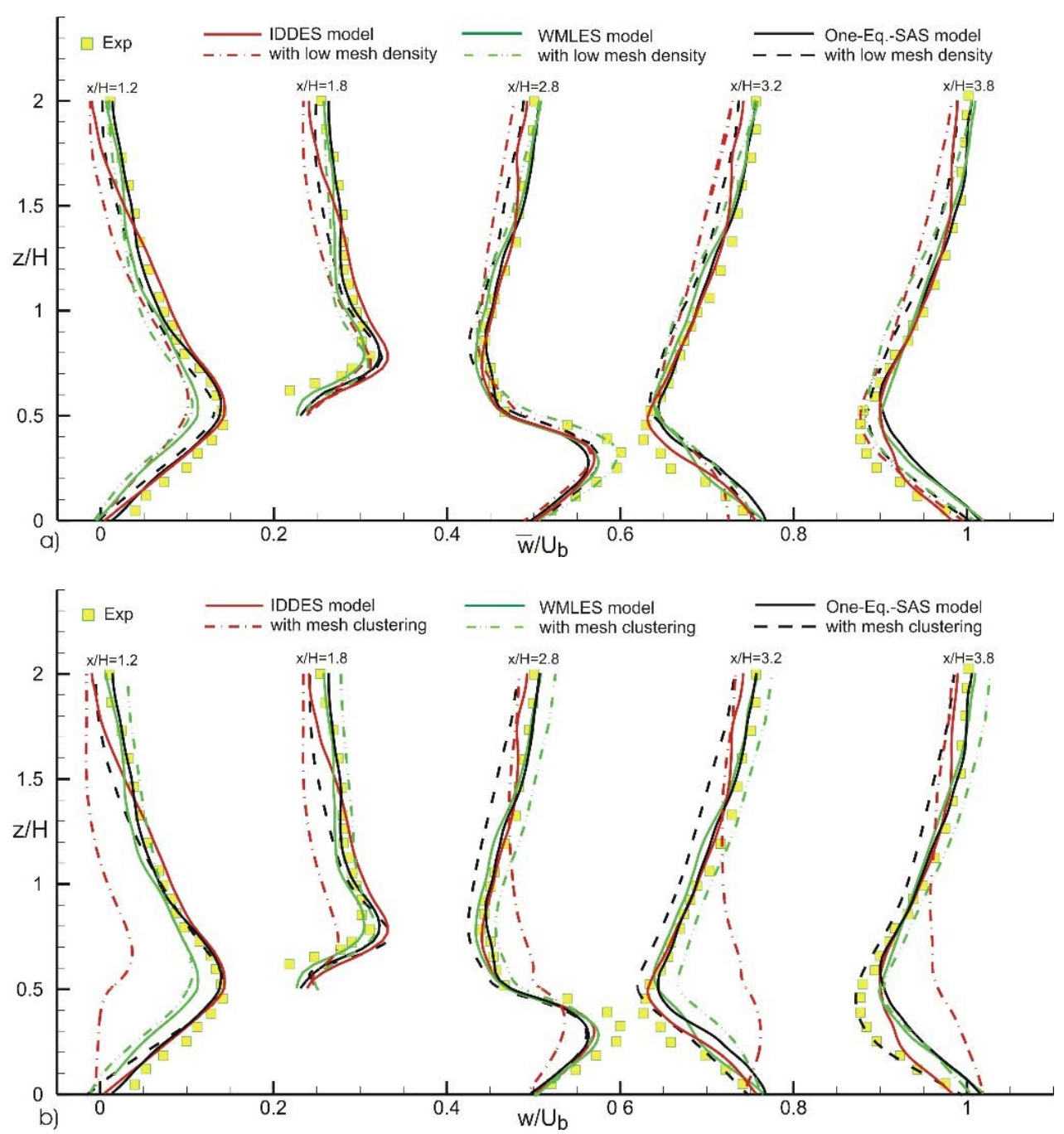

Figure 5: Mean spanwise velocity profiles on the horizontal $(x-z)$ plane that cuts through the center of the cube at $y / H=0.5$ showing effect of (a) mesh density; and (b) mesh clustering. Each successive $x / H$ profile starting from the one at $x / H=1.2$ is offset by 0.25 units from the previous profile.

between the cubes, i.e. $1.0<y / H<2.5$ as depicted in Fig. 6(a). When considering the effect of mesh clustering in Fig. 6(b) however, the One-Eq.-SAS model fairly reproduces the profiles. The IDDES model suffers the most and underpredicts the Reynolds normal stress in the region between $0<y / H<1.0$.

The same components of the Reynolds normal stress plotted on the $\mathrm{x}-\mathrm{z}$ horizontal plane at $y / H=0.5$ are depicted in Fig. 7. All models are able to predict the Reynolds normal stress profiles on both the coarse and regular mesh. However, the IDDES model predicts low levels in the circulatory region in between the cubes $(0.0<\mathrm{z} / \mathrm{H}<1.0)$ when comparing results of regular and clustered meshes. 

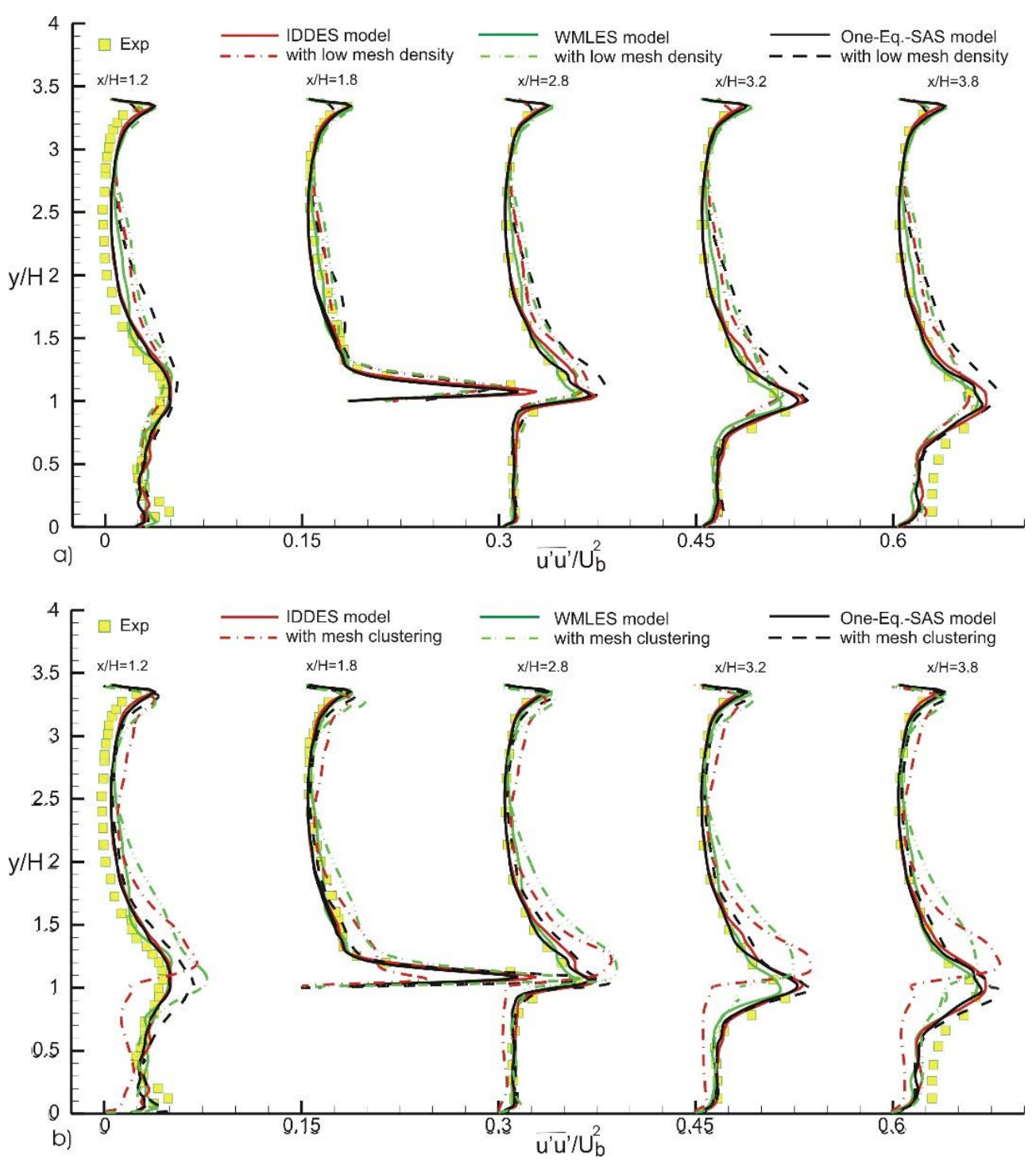

Figure 6: Streamwise normalized Reynolds stress profiles on the vertical (x-y) plane that cuts through the center of the cube at $\mathrm{z} / \mathrm{H}=0$ showing effect of (a) mesh density; and (b) mesh clustering.

Fig. 8 shows a comparison of spanwise normalized Reynolds normal stress, $\overline{w^{\prime} w^{\prime}} / U_{b}^{2}$, on the $x-y$ plane at the centerline of the cubes at $z / H=0$. While all models predict close distribution of Reynolds normal stress on both the coarse and regular meshes up to $x / H=2.8$, deviations are observed at $x / H=3.2$ and 3.8. The One-Eq.-SAS model provides the best conformance with experimental data at these two locations. The WMLES and the IDDES models do not accurately resolve profiles of the Reynolds normal stress, which is thought to 

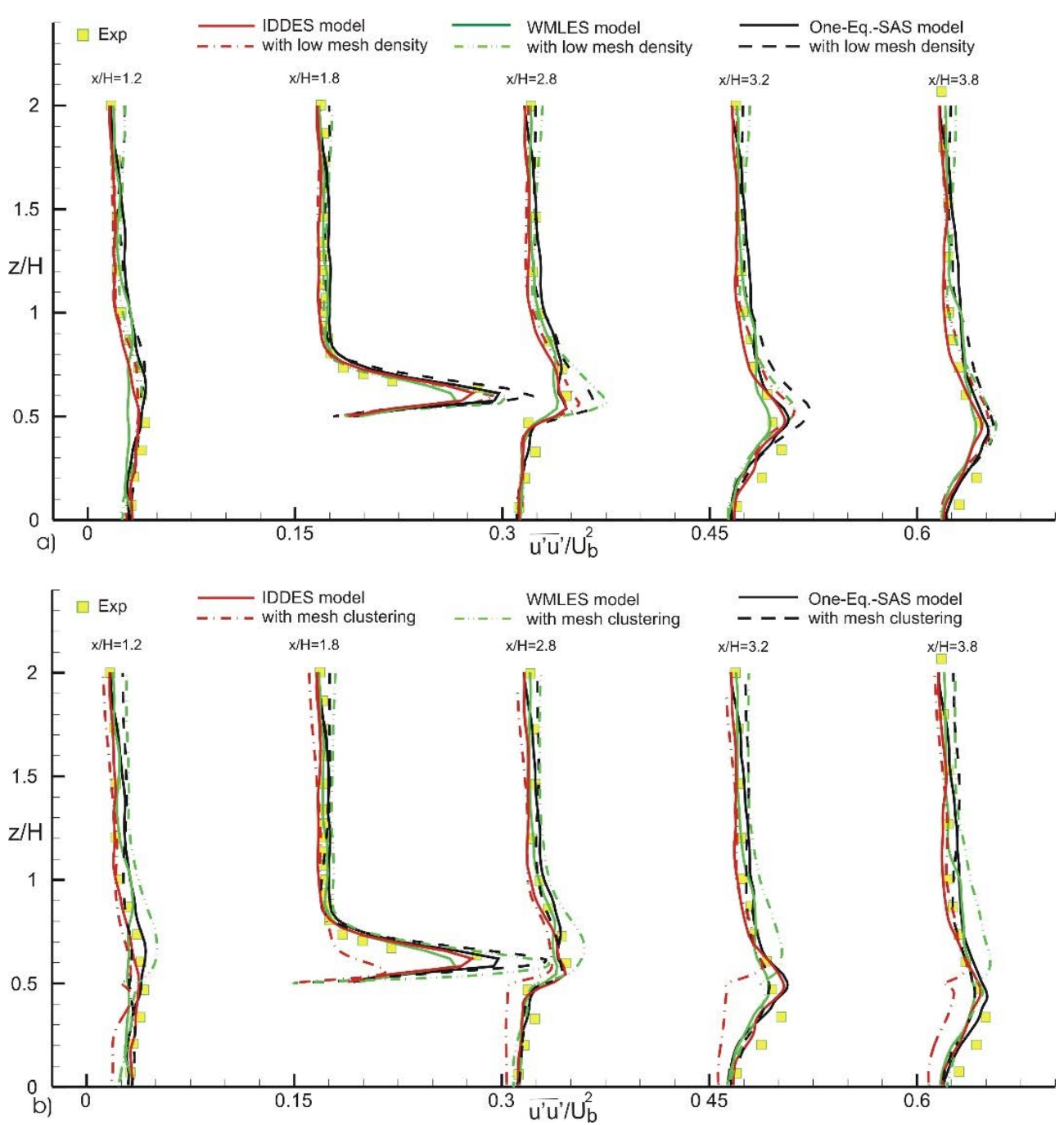

Figure 7: Streamwise normalized Reynolds stress profiles on the horizontal $(x-z)$ plane that cuts through the center of the cube and $y / H=0.5$ showing effect of (a) mesh density; and (b) mesh clustering.

be due to their inability to accurately model the subgrid stress. This deficiency seems to escalate when considering profiles of Reynolds normal stress in Fig. 8(b). while the One-Eq.SAS model is relatively unaffected by the clustering/ grid refinement issue, both the WMLES and the IDDES models exhibit noticeable discrepancies. This in turn is mainly due to wave reflect/unphysical perturbations that occurs as a result of grid-refinement process.

Profiles of normalized Reynolds normal stress plotted on the $\mathrm{x}-\mathrm{z}$ horizontal plane at $y / H=0.5$ are depicted in Fig. 9. Again models predict fair profiles for $x / H<2.8$ and deviate at $x / H=3.2$ and 3.8 as shown in Fig. 9(a). Both the WMLES and the IDDES models underpredict 

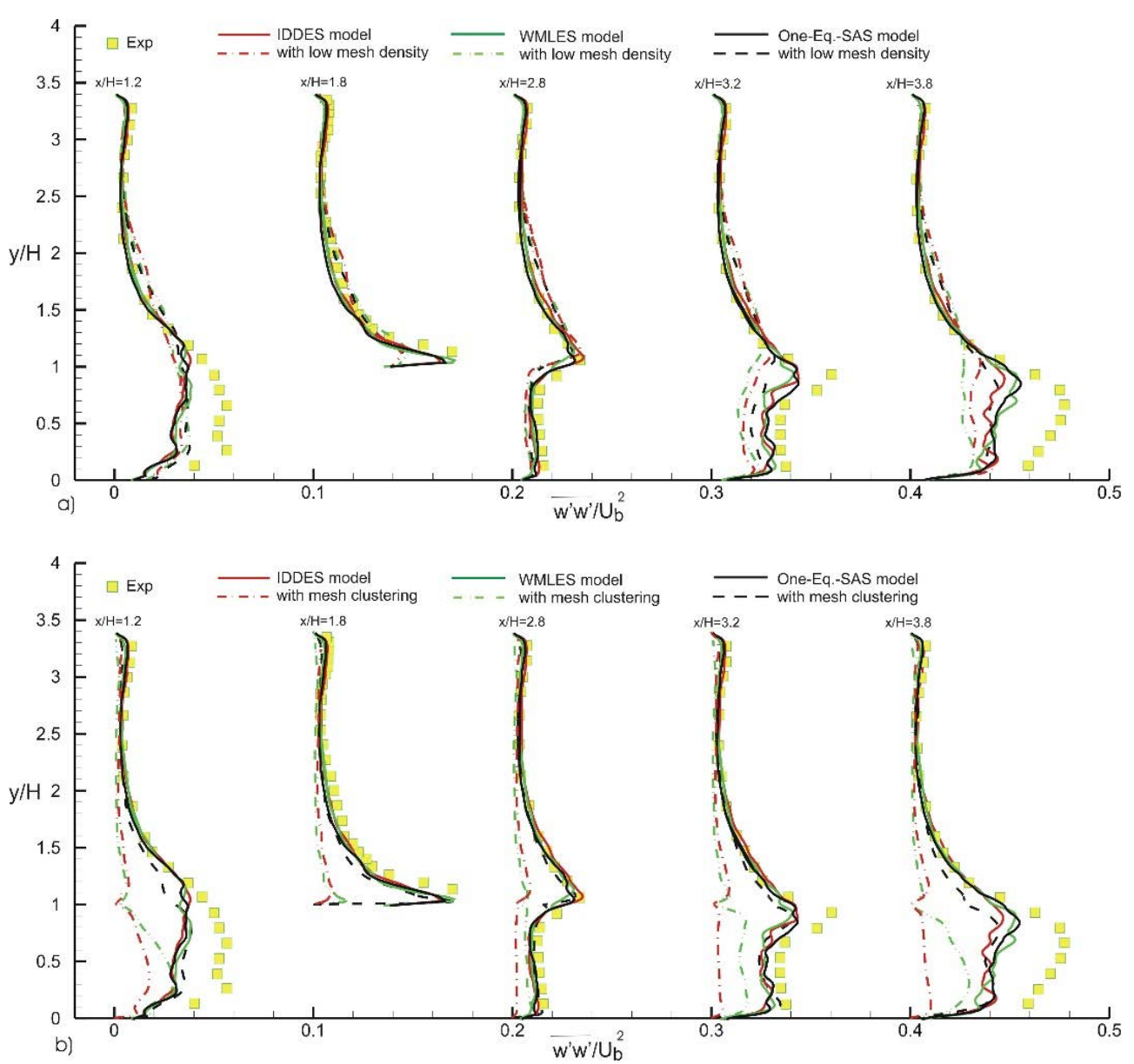

Figure 8: Spanwise normalized Reynolds stress profiles on the vertical $(x-y)$ plane that cuts through the center of the cube at $\mathrm{z} / \mathrm{H}=0$ showing effect of (a) mesh density; and (b) mesh clustering.

the Reynolds normal stress in circulatory region when simulations are carried out on a coarse grid compared to the One-Eq.-SAS model. The effect of grid-refinement however, is noticeably significant (Fig. 9(b)). The IDDES and the WMLES models fail to predict correct levels of Reynolds normal stress in the canyon street region and perform very poorly in the circulatory region between the cubes $(0.0<\mathrm{z} / \mathrm{H}<1.0)$, with the latter being more accurate than the former model. The One-Eq.-SAS model exhibits the least sensibility to variations in gridrefinement and grid density.

\section{CONCLUSION}

Three turbulence models have been examined over a periodic array of cubes, which is globally unstable involving a massive flow separation. All three considered models operate in SRS mode with the main difference that the WMLES and the IDDES models rely on grid 

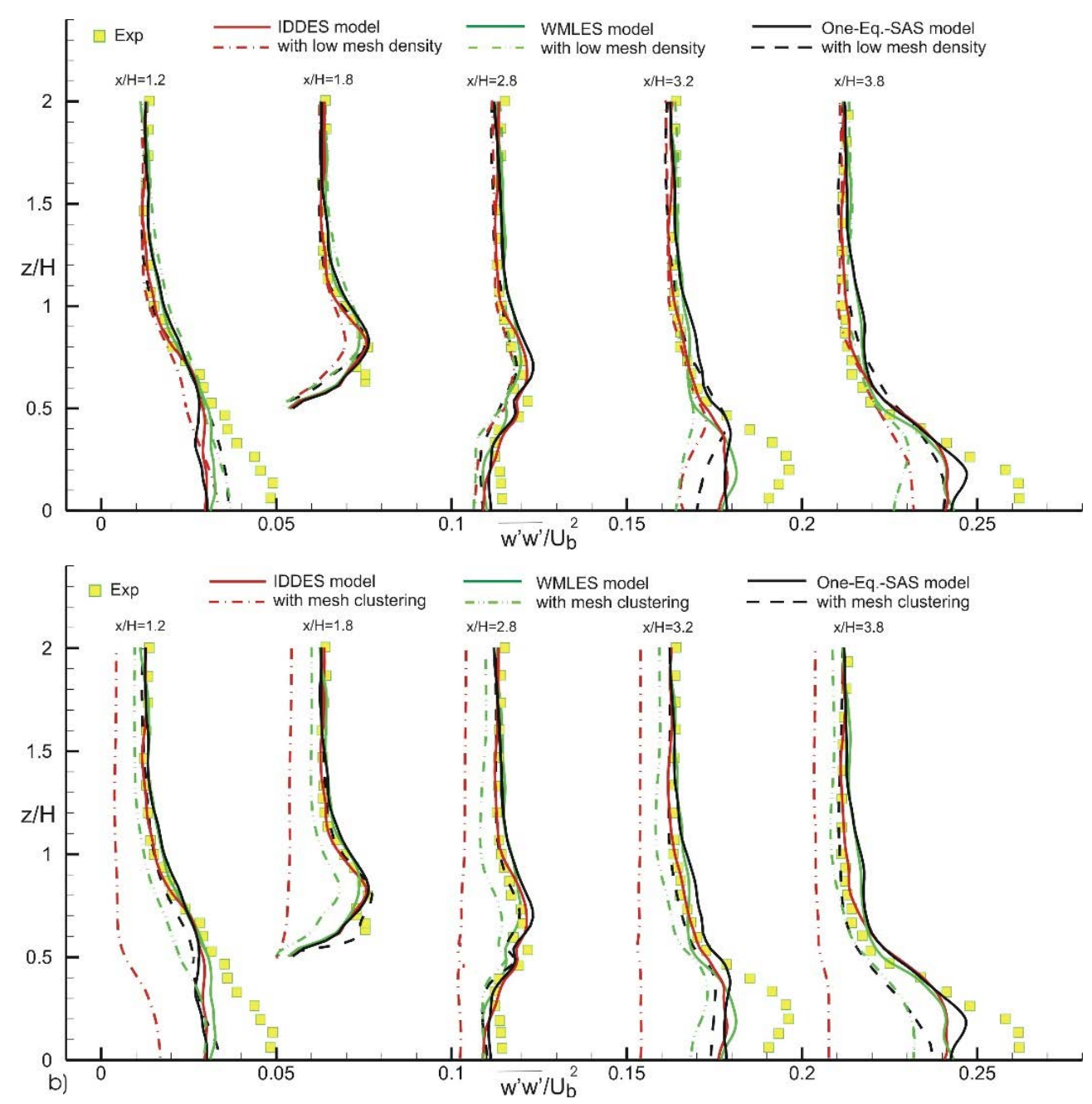

Figure 9: Spanwise normalized Reynolds stress profiles on the horizontal (x-z) plane that cuts through the center of the cube and $y / H=0.5$ showing effect of (a) mesh density, and (b) mesh clustering.

scale while the One-Eq.-SAS model depend on the Von-Kármán length scale that allows the model to operate in a SAS mode. Simulations were run on three different grids: a coarse, a regular, and a clustered one. The One-Eq.-SAS model was able to produce turbulent flow structures similar to LES and DES without suffering from the explicit grid sensitivity. In particular, the One-Eq.-SAS model outperformed the other two models on clustered grids and showed a better sub-grid-scale modelling on the coarse mesh. In both of these scenarios, the One-Eq.-SAS model had relatively the best predictions of velocity and Reynolds normal stress profiles and was closely followed by WMLES model. Among the considered three models, the IDDES had the fastest convergence in terms of the lowest number of iterations per time-step, which could be related to elevated levels of dissipation compare to the other two models. This point merits further investigation in future work. 


\section{ACKNOWLEDGEMENT}

This work is partially supported by the Lebanese American University School of Engineering Research Council.

\section{REFERENCES}

[1] Menter, F.R. \& Egorov, Y., A scale-adaptive simulation model using two-equation models. AIAA Paper-2005-1095. Reno NV, 2005.

[2] Elkhoury, M., Assessment of turbulence models for the simulation of turbulent flows past bluff bodies. Journal of Wind Engineering and Industrial Aerodynamics, 154, pp. 10-20, 2016.

[3] Bradshaw, P., Ferriss, D.H. \& Atwell, N.P., Calculation of boundary layer development using the turbulent energy equation. J. Fluid Mechanics, 23, pp. 31-64, 1967.

[4] Elkhoury, M., Partially lagging one-equation turbulence model. AIAA Journal, 53(12), pp. 3661-3673, 2015. DOI: 10.2514/1.J054018.

[5] Gritskevich, M.S., Garbaruk, A.V., Schutze, J. \& Menter F.R., Development of DDES and IDDES formulations for the $k-\omega$ shear stress transport model. Flow, Turbulence and Combustion, 88(3), pp. 431-449, 2012.

[6] Shur, M.L., Spalart, P.R., Strelets, M.K. \& Travin, A.K., A hybrid RANS-LES approach with delayed-DES and wall-modelled LES capabilities. International Journal of Heat and Fluid Flow, 29(6), pp. 1638-1649, 2008.

[7] Goodfriend, E., Katopodes Chow, F., Vanella, M. \& Balaras, E., Large-eddy simulation of flow through an array of cubes with local grid refinement. BoundaryLayer Meteorol., 159, pp. 285-303, 2016. DOI: 10.1007/s10546-016-0128-y.

[8] Meinders, E.R., Experimental study of heat transfer in turbulent flows over wallmounted cubes. PhD thesis, Faculty of Applied Sciences, Delft University of Technology: Delft, Netherlands, 1998.

[9] Meinders, E.R. \& Hanjalic, K., Vortex structure and heat transfer in turbulent flowover a wall-mounted matrix of cubes. International Journal of Heat Fluid Flow, 20, pp. 255-267, 1999.

[10] Hsieh, K.J., Lien, F.S. \& Yee, E., Towards a unified turbulence simulation approach for wall-bounded flows. Flow, Turbulence and Combustion, 84, pp. 193-218, 2010.

[11] Yang, X.I., Sadique, J., Mittal, R. \& Meneveau, C., Integral wall model for large eddy simulations of wall-bounded turbulent flows. Physics of Fluids, 27(2), pp. 1-32, 2015. 